\title{
The Effect of Sweet Potato Virus Disease and its Viral Components on Gene Expression Levels in Sweetpotato
}

\author{
C.D. Kokkinos ${ }^{1}$ and C.A. Clark \\ Department of Plant Pathology and Crop Physiology, Louisiana State University Agricultural Center, \\ Baton Rouge, LA 70803 \\ C.E. McGregor ${ }^{1}$ and D.R. LaBonte ${ }^{2}$ \\ Department of Horticulture, Louisiana State University Agricultural Center, Baton Rouge, LA 70803
}

\begin{abstract}
AdDitional INDEX WORDs. Ipomoea batatas, virus synergism, gene expression profile, sweet potato feathery mottle virus, sweet potato chlorotic stunt virus

Abstract. Sweet potato virus disease (SPVD) is the most devastating disease of sweetpotato [Ipomoea batatas (L.) Lam.] globally. It is caused by the co-infection of plants with a potyvirus, sweet potato feathery mottle virus (SPFMV), and a crinivirus, sweet potato chlorotic stunt virus (SPCSV). In this study we report the use of cDNA microarrays, containing $\mathbf{2 7 6 5}$ features from sweetpotato leaf and storage root libraries, in an effort to assess the effect of this disease and its individual viral components on the gene expression profile of $I$. batatas cv. Beauregard. Expression analysis revealed that the number of differentially expressed genes $(P<0.05)$ in plants infected with SPFMV alone and SPCSV alone compared to virus-tested (VT) plants was only 3 and 14, respectively. However, these findings are in contrast with SPVD-affected plants where more than $\mathbf{2 0 0}$ genes were found to be differentially expressed. SPVD-responsive genes are involved in a variety of cellular processes including several that were identified as pathogenesis- or stress-induced.
\end{abstract}

Sweetpotato is the seventh most important food crop in the world, with annual world production of $\approx 130$ million tonnes. It ranks third among root and tuber crops worldwide (Food and Agriculture Organization of the United Nations, 2005). Viral diseases, including those caused by mixed infections, are of major economic importance in most production areas around the globe. The use of vegetative cuttings as a principal propagation method provides viruses an efficient way to perpetuate and disseminate between growing seasons as well as growing areas (Salazar and Fuentes, 2001). As many as 19 different viruses have been identified in sweetpotato and 11 of these are currently recognized by the International Committee of Taxonomy of Viruses (Kreuze, 2002). The effects of these viruses on production range from minimal, to completely devastating, depending on the infecting virus, virus complexes, and sweetpotato cultivars involved.

The most important and devastating viral disease affecting sweetpotatoes worldwide is sweet potato virus disease (SPVD). Yield losses of up to $90 \%$ have been reported in plants affected with SPVD (Gutiérrez et al., 2003; Hahn, 1976; Ngeve, 1990). SPVD is caused by a synergistic interaction between a potyvirus, sweet potato feathery mottle virus (SPFMV), and a crinivirus,

Received for publication 7 Feb. 2006. Accepted for publication 25 May 2006. Approved for publication by the director of the Louisiana Agricultural Experiment Station as manuscript number 05-38-0752. The authors would like to thank Bryon Sosinski (Dept. of Horticultural Science, North Carolina State Univ., Raleigh) and Kornel Burg (ARC Seibersdorf research GmbH, A-2444 Seibersdorf, Austria) for making the cDNA clones available, Limei He, Regina Ali (both from CALS Genome Research Lab, North Carolina State University, Raleigh NC 27695, USA) and Joanna Jankowicz (ARC Seibersdorf research $\mathrm{GmbH}$ ) for their hard work during development of the sweetpotato microarray, and Silvia Fluch (ARC Seibersdorf research $\mathrm{GmbH}$ ) for printing the arrays. We would also like to thank Dr. R.A. Valverde for critically reviewing this manuscript. This research was supported in part by funds from the McKnight Foundation Collaborative Crop Research Program.

These authors contributed equally to this research.

${ }^{2}$ Corresponding author: Don R. LaBonte; Telephone: +225-578-1024; Fax: +225578-1068; E-mail: DLabonte@agcenter.1su.edu sweet potato chlorotic stunt virus (SPCSV). Plants co-infected with SPFMV or other sweetpotato potyviruses and SPCSV exhibit severe symptoms such as leaf strapping, vein clearing, leaf distortion, chlorosis, puckering, and stunting. The severity of symptoms, which develop first in the newly emerging leaves, can be directly associated with the dramatic yield reductions observed (Salazar and Fuentes, 2001). The time from initial infection to the appearance of symptoms varies depending on age and size of the plant, with symptoms taking longer to develop on older and bigger plants (Karyeija et al., 2000). SPVD has been reported in a number of African countries, including Rwanda, Burundi, Uganda, Ghana, Nigeria, Kenya, Tanzania, Zimbabwe (reviewed by Karyeija et al., 1998a), and Egypt (Ishak et al., 2003). Outside Africa, this disease has been reported in Israel (Loebenstein and Harpaz, 1960), Spain (Valverde et al., 2004), and Peru (Gutierrez et al., 2003). Since SPFMV is found wherever sweetpotatoes are grown and SPCSV has recently been reported in China (Zhang et al., 2005) and Korea (Yun et al., 2002), SPVD is thus likely to occur in these countries as well. In Argentina, a similar synergism, known as chlorotic dwarf, has been reported that also includes a third virus, sweet potato mild speckling virus (Di Feo et al., 2000).

SPFMV, a member of the Potyviridae family and the Potyvirus group, is transmitted by a number of aphid species, including Aphis gossypii Glover and Myzus persicae Sulzer. Plants infected with SPFMV alone, often are symptomless or exhibit mild symptoms and the yield losses are usually minimal (Clark and Hoy, 2006; Gutiérrez et al., 2003). The titers of SPFMV in these plants are similarly low (Kokkinos and Clark, 2006). However, the titers increase dramatically when plants are co-infected with SPCSV (Karyeija et al., 2000; Kokkinos and Clark, 2004), with a corresponding increase in the severity of disease symptoms and yield loss. SPFMV is common wherever sweetpotatoes are grown (Brunt el al., 1996). In the U.S. two strains of SPFMV are recognized, the common strain (SPFMV-C) and the russet crack strain (SPFMV-RC). However, SPFMV-C does not cause 
typical SPVD symptoms in the presence of SPCSV. Symptoms are usually mild and transient or typical of single infections with SPCSV (Souto et al., 2003).

Infection of sweetpotatoes with the whitefly-transmitted (Bemisia tabaci Gennadius, Trialeurodes abutilonea Haldeman), phloem-limited crinivirus (family Closteroviridae) SPCSV alone can lead to mild to moderate symptoms, with yield losses of up to $43 \%$ (Gutiérrez et al., 2003). This virus consists of two distinct strain groups, the east African (EA) and west African (WA), both of which are able to cause synergistic disease (Ishak et al., 2003; Tairo 2005). The titers of this virus are relatively high in infected plants. Interestingly, the titers do not change significantly after co-infection with SPFMV (Karyeija et al., 2000). To date SPCSV has only been found in the United States, in a tissue culture accession and not in the field (Pio-Ribeiro et al., 1996).

Efforts to breed for resistance to SPVD have until now focused mainly on breeding for resistance to SPFMV and many sweetpotato cultivars are reasonably resistant to SPFMV (Gibson et al., 1998). Efforts to use SPFMV resistance to breed for SPVD resistance have been unsuccessful because the SPFMV resistance is broken when plants are co-infected with SPCSV (Karyeija et al., 1998b). The mechanism underlying the synergistic interaction between SPFMV and SPCSV and its effect on the host's response to infection are not known. It is possible that other molecular interactions in the dual infection process may provide better opportunities for resistance to SPVD than narrowly focusing on resistance to SPFMV. Understanding this phenomenon is essential if breeding for resistance to SPVD is going to be successful. An understanding of host-pathogen interactions on the molecular level can provide new insights into the effect of the synergism between SPFMV and SPCSV on the host, and can lead to new approaches in breeding for resistance to SPVD.

Microarray technology (Schena et al., 1995) makes possible the assessment of relative gene expression levels of thousands of genes simultaneously. Genes from the organism under investigation (sweetpotato in this case) are spotted on a glass slide, which is then hybridized with mRNA from different treatments. The use of two different florescent dyes makes it possible to hybridize two treatments (or a treatment and control) on a single array. After hybridization the array is scanned using a fluorescent scanner and computer software is used to extract intensity values from the image. Statistical analysis of the data makes it possible to determine which genes are differentially expressed between treatments. Microarrays have already been used to investigate host-pathogen interactions in plants (De Vos et al., 2005; Dowd et al., 2004; Gibly et al., 2004; Moy et al., 2004) and other organisms (for review see Kato-Maeda et al., 2001). Virus associated host-pathogen interactions have been studied in a range of organisms, from humans (Zhu et al., 1998) to Arabidopsis thaliana (L.) Heynh., (Golem and Culver, 2003; Whitham et al., 2003). In this paper we report the use of sweetpotato cDNA microarray technology in an effort to better understand the effect of the synergistic interaction between SPFMV and SPCSV on the host's response to infection. This study represents the first effort to investigate the effect of SPVD and its viral components on gene expression of sweetpotato.

\section{Materials and Methods}

Plant material and inoculations. Ipomoe a setosa Ker-Gawl. seedlings mechanically inoculated with SPFMV-RC (isolate 952 ), and I. batatas cv. Beauregard plants infected with SPCSV (isolate BWFT-3) alone were grown in the greenhouse to generate the scions that were used to graft-inoculate clonally propagated plants of virus-tested [VT plants are tested for presence of viruses by grafting three times to an indicator host, I. setosa] I. batatas cv. Beauregard. Test plants were graft-inoculated 3 weeks after planting. A single wedge graft per virus was performed and individuals on which the scion(s) survived for at least three weeks were selected and used in this study. The experiment consisted of the following four treatments in a randomized complete-block design: VT (not inoculated), SPFMV-RC (VT plants graft inoculated with SPFMV-RC alone), SPCSV (VT plants graft inoculated with SPCSV alone) and SPVD (VT plants graft inoculated with SPFMV-RC and SPCSV simultaneously). Each treatment was replicated six times. Plants were grown under standard greenhouse conditions in 15-cm-diameter clay pots containing autoclaved soil mix consisting of 1 part river silt, 1 part sand, 1 part Jiffy-Mix Plus (Jiffy Products of America, Norwalk, Ohio) and $3.5 \mathrm{~g}$ per pot of Osmocote 14N-6.1P-11.6K (Scotts-Sierra Horticultural Products Co., Marysville, Ohio). A weekly insecticide spray program was used to control aphids and whiteflies. At 9 weeks after inoculation the first four fully opened leaves from the top of each test plant were collected, combined and immediately frozen in liquid nitrogen and stored at $-80^{\circ} \mathrm{C}$ until extraction. Nine weeks after inoculation was selected as the collection date to ensure better uniformity in virus titers (Kokkinos and Clark, 2006) and symptom development between biological replicates.

RNA ISOLATION, LABELING, AND ARRAY HYBRIDIZATION. Total RNA was extracted from six plants of each treatment. After leaf materials were ground with a mortar and pestle in liquid nitrogen, $\approx 0.8 \mathrm{~g}$ were used to extract total RNA using the RNeasy Maxi Kit (Qiagen, Valencia, Calif.) according to the manufacturer's instructions. The RNA was further cleaned and concentrated by using the clean-up procedure as described in the RNAeasy Mini Kit Manual (Qiagen). During both steps, DNase I digestion was carried out on the column as recommended by the manufacturer.

For each sample, $10 \mu \mathrm{g}$ of total RNA was labeled using the SuperScript Indirect cDNA Labeling System for DNA Microarrays (Invitrogen, Carlsbad, Calif.) according to the manufacturer's protocol. Samples were labeled with $\mathrm{Cy} 3$ or Cy 5 fluorescent labels (Amersham Biosciences, Piscataway, N.J.) and hybridized onto arrays in a connected loop design. (Rosa et al., 2005) using the Pronto hybridization kit (Corning, Life Sciences, Corning, N.Y.). To limit dye effects, the order of the treatments in the loops, as well as the direction of labeling were varied. The order of samples in the loops and the direction of the labeling were different for different loops to ensure that a specific comparison in the loop is not always labeled with the same dye and hybridized together on the same array.

ArRAYARCS_SP02/2.The sweetpotatoARCS_SP02/02 array contains 3600 features, spotted in triplicate with a Genemachines Omnigrid microarray printer (GeneMachines, San Carlos, Calif.) on Corning GAPSII slides (Corning Inc.). The arrays were printed and supplied by S. Fluch at ARC Seibersdorf Research GmbH (Biogenetics/Natural Resources, Seibersdorf, Austria). The array contains 2765 features from sweetpotato leaf and sweetpotato storage root libraries as well as control features, including nonplant features, spotting buffer features and blanks. The sequence information for the sweetpotato cDNAs features spotted on the array is available online in GenBank.

Array scanning, image Quantification, and statistical ANALYSIS. Arrays were scanned with an AlphaArray Reader (Alpha Innotech, San Leandro, Calif.) and spots were detected and 
quantified using UCSF Spot (Jian et al., 2002). After comparing the effects of different normalization methods using MA-plots (the intensity log-ratio, M vs. the mean log intensity) (Dudoit et al., 2002), and spatial image plots, data were normalized within (printtip loess) (Smyth and Speed, 2003) and between slides (scaled). Linear models (Smyth, 2004) were fitted for comparisons between treatments and genes were considered differentially expressed if $P$ $<0.05$ after applying the Holm (1979) multiple testing correction. All normalizations and statistical analyses were carried out using limmaGUI software (Wettenhall and Smyth, 2004). In this study, the output from limmaGUI is in the form of M-values $\left(\log _{2}\right.$ fold change) (Wettenhall and Smyth, 2004) (Table 1).

QuANTITATIVE REAL-TIME POLYMERASE CHAIN REACTION (QRT-PCR). Two-step Q-RT-PCR was carried out for seven genes using RNA from the six VT and six SPVD affected plants. Firststrand cDNA synthesis was carried out using the SuperScript III First-Strand Synthesis System for RT-PCR (Invitrogen) and the resulting product was diluted by adding $40 \mu \mathrm{L}$ water. One microliter of the dilution was used for Q-RT-PCR on the ABI PRISM 7000 Sequence Detection System using SYBR Green PCR Master Mix (Applied Biosystems, Foster City, Calif.) and $600 \mathrm{~nm}$ of each primer (Table 2) in a final volume of $25 \mu \mathrm{L}$. The following PCR protocol was followed: $95^{\circ} \mathrm{C}$ for $10 \mathrm{~min}$, followed by 40 cycles of $95^{\circ} \mathrm{C}$ for $15 \mathrm{~s}$ and $55^{\circ} \mathrm{C}$ for $1 \mathrm{~min}$. Amplifications from $18 \mathrm{~S}$ ribosomal RNA specific primers (Applied Biosystems) were used to normalize data and dissociation curves were used to detect nonspecific amplification. Significant differences $(P<$ 0.05 ) between treatments were determined using a $t$ test (variances not assumed equal) of normalized values.

FunCTIONAL CLASSIFICATION OF GENES. Gene descriptions were obtained by comparison of sequences to GenBank and $A$. thaliana protein sequences (TIGR) (BLASTXE-value < 1E-5). Functional classification of genes in Table 1 was based on information from the Munich Information Center for Protein Sequences (Schoof et al., 2002).

\section{Results and Discussion}

The number of genes differentially expressed between VT plants and the three treatments varied. Between VT and SPFMVRC, and VT and SPCSV, only 3 and 14 genes were differentially expressed, respectively, compared to 216 between VT and SPVD (Table 1). The number of differentially expressed genes was analogous to the severity of symptoms observed in the three viral treatments. At the time leaf samples were collected from SPFMV-RC-infected plants, and throughout the time period between inoculation and sample collection, no symptoms were observed, typical of single potyvirus infections (presence of the virus was confirmed by grafting of scions from test plants to $I$. setosa). Symptoms of SPCSV-infected plants at the time of collection however, were distinct and characteristic of SPCSV single infections and included interveinal chlorosis and mild purpling. As expected, the most severe symptoms were observed with SPVDaffected plants, which exhibited vein clearing, leaf distortion, chlorosis, puckering, and overall stunting. When comparing VT plants and plants infected with SPCSV alone, only 3 of the 14 differentially expressed genes were suppressed by SPCSV. One of these genes, plastocyanin, was suppressed in all virus-infected treatments. Of the 216 genes differentially expressed between VT and SPVD affected plants, 93 genes were induced in SPVD and 123 suppressed. Many of the genes suppressed in SPVD affected plants are related to photosynthesis and metabolism. Of the induced genes many are involved in protein synthesis and protein fate (Table 1).

Q-RT-PCR analysis was carried out for seven genes determined to be differentially expressed between VT and SPVD affected plants by microarray analysis. The results indicated that all seven genes were also significantly differentially expressed $(P<0.05)$ using Q-RT-PCR with comparable fold changes (Table 3). This reinforces our assumptions regarding significant differential expression based on limmaGUI analyses.

During their infection cycles, viruses need plant proteins for accumulation and movement. Gene expression in the host is affected by virus infection. The host plant can respond to an infection by activating specific or general resistance pathways (Whitham etal., 2003). By determining which genes are differentially expressed in the host during infection, we hope to elucidate how the response of sweetpotato plants to dual infections of SPFMV and SPCSV differs from response to single infections.

The reduction of expression levels of genes that are directly or indirectly involved in the overall photosynthetic pathway, clearly observed in the SPVD-affected plants in this study, is a phenomenon commonly observed in yellows diseases and leaves of plants showing typical chlorotic or mosaic symptoms as a result of virus infection (Hull, 2002). Our data support previous reports, which indicate that the reduction in photosynthesis, observed in virus infected plants, is correlated with the reduction of photosynthetic pigments, rubisco, and specific proteins associated with photosystem II (Naidu et al., 1986; van Kooten et al., 1990) and reduced activity of the crassulacean acid metabolism (CAM) (Izaguirre-Mayoral et al., 1993). As expected, the effect on expression levels of "photosynthetic" genes in plants infected with either SPFMV or SPCSV alone was minimal since these viruses, when infecting this particular sweetpotato cultivar alone, cause mild and transient symptoms.

Plant resistance genes ( $R$ genes) are able to recognize pathogens carrying the corresponding avirulence genes (gene-for-gene resistance). This recognition triggers the hypersensitive response (HR), which includes programmed cell death (PCD). The HR is often preceded by the accumulation and production of reactive oxygen species (ROS), including hydrogen peroxide $\left(\mathrm{H}_{2} \mathrm{O}_{2}\right)$ (Glazebrook, 2001). Several genes, which were differentially expressed only in plants affected by SPVD, were identified as resistance-related or stress-induced genes. Interestingly, some of these genes were down-regulated whereas others were up-regulated. Two putative $R$ genes, one belonging to the TIR-NBS-LRR class (DV036322) and the other belonging to the CC-NBS-LRR class (DV035471) were induced in SPVD affected plants. A NDR1/HIN1-like (CB330891) gene, known to be required by most CC-NBS-LRR class resistance genes in A. thaliana (Aarts et al., 1998) was also induced in SPVD. DV036322 shows homology to At5g17680.1 of A. thaliana, while DV035471 is homologous to At1 g58602.1. These genes are similar to ones which encode known disease resistance proteins rpp8 and RPP1-WsB, respectively. To our knowledge, no $R$ genes have been reported, nor is there previous evidence for gene-for-gene resistance in sweetpotato. It is probable that these two genes play some other role in sweetpotato, possibly in apoptosis or ATP-binding.

One of the genes found to be down-regulated in SPVD, encodes a product belonging to the ankyrin repeat-containing protein family (DV036499). In transformed $A$. thaliana, an ankyrin repeat-containing protein was found to be directly associated with the oxidative metabolism of the host's resistance to disease and stress response (Yan et al., 2002). The down-regulation of ankyrin 
Table 1. Selected genes in sweetpotato differentially expressed $(P<0.05)$ between virus-tested $(\mathrm{VT})$, sweet potato feathery mottle virus russet crack strain-infected (SPFMV), sweet potato chlorotic stunt virus-infected (SPCSV), and plants infected with SPFMV and SPCSV (SPVD).

\begin{tabular}{|c|c|c|c|c|c|c|c|c|}
\hline \multirow[b]{2}{*}{$\begin{array}{l}\text { GenBank } \\
\text { accession no. }\end{array}$} & \multirow[b]{2}{*}{ Gene description } & \multirow[b]{2}{*}{$\begin{array}{c}\text { E- } \\
\text { value }^{\mathrm{z}}\end{array}$} & \multicolumn{6}{|c|}{ M-values ${ }^{\mathrm{y}}$} \\
\hline & & & $\begin{array}{l}\mathrm{VT}^{\mathrm{x}}- \\
\text { SPFMV }\end{array}$ & $\begin{array}{l}\text { VT }^{\mathrm{x}}- \\
\text { SPCSV }\end{array}$ & $\begin{array}{l}V^{x}- \\
\text { SPVD }\end{array}$ & $\begin{array}{l}\text { SPFMV } \\
\text {-SPCSV }\end{array}$ & $\begin{array}{l}\text { SPFMV } \\
\text {-SPVD } \\
\end{array}$ & $\begin{array}{l}\text { SPCSV } \\
\text {-SPVD } \\
\end{array}$ \\
\hline \multicolumn{9}{|c|}{ Cell rescue, defense, and virulence } \\
\hline CB330627 & Bet v I allergen family protein & $4 \mathrm{E}-24$ & & & 0.68 & & 0.63 & 0.50 \\
\hline DV036659 & catalase 2 & $1 \mathrm{E}-103$ & & & -0.62 & & & \\
\hline DV035471 & $\begin{array}{l}\text { disease resistance protein (CC-NBS- } \\
\text { LRR class) }\end{array}$ & $6 \mathrm{E}-08$ & & & -0.40 & & & -0.43 \\
\hline DV036322. & $\begin{array}{l}\text { disease resistance protein (TIR-NBS- } \\
\text { LRR class) }\end{array}$ & $4 \mathrm{E}-31$ & & & -0.47 & & -0.44 & -0.48 \\
\hline CB330666 & metallothionein-like type 1 protein & $3 \mathrm{E}-16$ & -0.46 & -0.76 & -0.89 & & -0.42 & -0.46 \\
\hline CB330120 & $\begin{array}{l}\text { metallothionein protein, putative } \\
\text { (MT2A) }\end{array}$ & $4 \mathrm{E}-06$ & & & 0.43 & & & \\
\hline CB330891 & NDR1/HIN1-like protein & $8 \mathrm{E}-10$ & & & -1.02 & & -0.97 & -0.89 \\
\hline CB330630 & $\begin{array}{l}\text { peroxidase } 42 \text { (PER42) (P42) } \\
\text { (PRXR1) }\end{array}$ & $2 \mathrm{E}-27$ & & & 0.55 & & 0.48 & \\
\hline CB330206 & $\begin{array}{l}\text { Rac-like GTP-binding protein } \\
\text { (ARAC10) }\end{array}$ & $5 \mathrm{E}-89$ & & & 0.72 & & 0.71 & 0.52 \\
\hline CB330564 & $\begin{array}{l}\text { trigger factor type chaperone family } \\
\text { protein }\end{array}$ & $5 \mathrm{E}-98$ & & & 0.72 & & 0.77 & \\
\hline \multicolumn{9}{|c|}{ Protein synthesis and protein fate } \\
\hline DV035469 & $\begin{array}{l}\text { 20S proteasome beta subunit } \mathrm{E} \text {, } \\
\text { putative }\end{array}$ & $5 \mathrm{E}-31$ & & & -0.61 & & -0.68 & \\
\hline DV034935 & $\begin{array}{l}\text { 30S ribosomal protein } \mathrm{S} 13 \text {, } \\
\text { chloroplast (CS13) ribosomal protein }\end{array}$ & $3 \mathrm{E}-50$ & & & -0.56 & & & \\
\hline & S13 precursor & & & & & & & \\
\hline DV034886 & 40S ribosomal protein S3 (RPS3C) & $1 \mathrm{E}-104$ & & & -0.79 & & -0.69 & -0.71 \\
\hline DV037420 & 40S ribosomal protein S10 (RPS10C) & $1 \mathrm{E}-48$ & & & -0.50 & & -0.52 & -0.44 \\
\hline DV037214 & $\begin{array}{l}\text { 60S ribosomal protein L13A } \\
\text { (RPL13aB) }\end{array}$ & $1 \mathrm{E}-107$ & & -1.08 & & -1.14 & & \\
\hline CB330735 & $\begin{array}{l}\text { 60S ribosomal protein } \mathrm{L} 26 \\
\text { (RPL26A) }\end{array}$ & $1 \mathrm{E}-48$ & & & -0.74 & & -0.73 & -0.68 \\
\hline DV036489 & $\begin{array}{l}\text { 60S ribosomal protein } \mathrm{L} 31 \\
\text { (RPL31A) }\end{array}$ & $9 \mathrm{E}-40$ & & & 0.37 & & 0.39 & 0.41 \\
\hline CB330088 & $60 \mathrm{~S}$ ribosomal protein L36a/L44 & $2 \mathrm{E}-45$ & & & -0.80 & & -0.73 & -0.74 \\
\hline CB330146 & $\begin{array}{l}\text { elongation factor } 1 \mathrm{~B} \text {-gamma, } \\
\text { putative / eEF-1B gamma, putative }\end{array}$ & $2 \mathrm{E}-48$ & & & 0.65 & & 0.59 & 0.48 \\
\hline CB329890 & $\begin{array}{l}\text { eukaryotic translation initiation } \\
\text { factor } 2 \mathrm{~B} \text { family protein / eIF-2B } \\
\text { family protein }\end{array}$ & $1 \mathrm{E}-136$ & & & 0.54 & & 0.48 & \\
\hline CB330048 & cyclophilin-type family protein & $5 \mathrm{E}-42$ & & & -0.61 & & -0.66 & -0.63 \\
\hline CB330102 & $\begin{array}{l}\text { polyubiquitin (UBQ10) (SEN3) } \\
\text { senescence-associated protein }\end{array}$ & $2 \mathrm{E}-77$ & & & -0.53 & & -0.77 & -0.52 \\
\hline CB330070 & subtilase family protein & $1 \mathrm{E}-35$ & & & 0.77 & & 0.63 & 0.54 \\
\hline \multicolumn{9}{|l|}{ Metabolism } \\
\hline CB330699 & $\begin{array}{l}\text { adenine phosphoribosyltransferase, } \\
\text { putative }\end{array}$ & $1 \mathrm{E}-83$ & & & 0.36 & & 0.35 & \\
\hline DV037724 & adenosine kinase 2 (ADK2) & $8 \mathrm{E}-83$ & & & -0.42 & & & \\
\hline CB330084 & $\begin{array}{l}\text { cinnamyl-alcohol dehydrogenase } \\
\text { (CAD), putative }\end{array}$ & $1 \mathrm{E}-104$ & & & 0.33 & & & 0.36 \\
\hline CB330293 & $\begin{array}{l}\text { coproporphyrinogen III oxidase, } \\
\text { putative }\end{array}$ & $3 \mathrm{E}-45$ & & & 1.03 & & 0.73 & 0.67 \\
\hline DV037506 & $\begin{array}{l}\text { eukaryotic translation initiation } \\
\text { factor } 5 \mathrm{~A}-1 \text { (eIF-5A 1) }\end{array}$ & $4 \mathrm{E}-59$ & & & -0.50 & & -0.57 & -0.46 \\
\hline CB330285 & $\begin{array}{l}\text { ferredoxin-thioredoxin reductase, } \\
\text { putative }\end{array}$ & $5 \mathrm{E}-27$ & & & 0.66 & & 0.75 & 0.67 \\
\hline
\end{tabular}


Table 1. Continued.

\begin{tabular}{|c|c|c|c|c|c|c|c|c|}
\hline \multirow[b]{2}{*}{$\begin{array}{l}\text { GenBank } \\
\text { accession no. }\end{array}$} & \multirow[b]{2}{*}{ Gene description } & \multirow[b]{2}{*}{$\begin{array}{c}\text { E- } \\
\text { value }^{z}\end{array}$} & \multicolumn{6}{|c|}{ M-values ${ }^{y}$} \\
\hline & & & $\begin{array}{l}\text { VT }^{\mathrm{x}}- \\
\text { SPFMV }\end{array}$ & $\begin{array}{l}\mathrm{VT}^{\mathrm{x}}- \\
\text { SPCSV }\end{array}$ & $\begin{array}{l}V^{\mathrm{x}}- \\
\text { SPVD }\end{array}$ & $\begin{array}{l}\text { SPFMV } \\
\text {-SPCSV }\end{array}$ & $\begin{array}{l}\text { SPFMV } \\
\text {-SPVD }\end{array}$ & $\begin{array}{l}\text { SPCSV } \\
\text {-SPVD }\end{array}$ \\
\hline CB330640 & $\begin{array}{l}\text { fructose-bisphosphate aldolase, } \\
\text { putative }\end{array}$ & $3 \mathrm{E}-64$ & & & 0.90 & & 0.96 & 1.23 \\
\hline CB329981 & $\begin{array}{l}\text { glucose-6-phosphate isomerase, } \\
\text { putative }\end{array}$ & $6 \mathrm{E}-86$ & & & 0.55 & & & \\
\hline CB330405 & $\begin{array}{l}\text { glutamate:glyoxylate } \\
\text { aminotransferase } 2 \text { (GGT2) }\end{array}$ & $5 \mathrm{E}-32$ & & & 0.49 & & & \\
\hline CB330166 & $\begin{array}{l}\text { glyceraldehyde-3-phosphate } \\
\text { dehydrogenase, cytosolic (GAPC) }\end{array}$ & $1 \mathrm{E}-159$ & & & 0.48 & & & \\
\hline CB330355 & $\begin{array}{l}\text { glycine cleavage system } \mathrm{H} \text { protein, } \\
\text { mitochondrial, putative }\end{array}$ & $7 \mathrm{E}-25$ & & & 0.60 & & 0.59 & \\
\hline CB330544 & $\begin{array}{l}\text { phosphoglycolate phosphatase, } \\
\text { putative }\end{array}$ & $2 \mathrm{E}-75$ & & & 0.68 & & 0.55 & 0.55 \\
\hline CB330622 & $\begin{array}{l}\text { ribulose bisphosphate carboxylase } \\
\text { small chain } 2 \mathrm{~B} / \mathrm{RuBisCO} \text { small } \\
\text { subunit } 2 \mathrm{~B} \text { (RBCS-2B) (ATS2B) }\end{array}$ & $3 \mathrm{E}-62$ & & & 1.15 & & 1.05 & 1.34 \\
\hline DV035761 & $\begin{array}{l}\text { shaggy-related protein kinase kappa / } \\
\text { ASK-kappa (ASK10) }\end{array}$ & $7 \mathrm{E}-57$ & & & 0.76 & & 0.59 & 0.56 \\
\hline DV037227 & sterol desaturase family protein & $7 \mathrm{E}-58$ & & & -0.55 & & -0.56 & -0.54 \\
\hline CB330375 & terpene synthase/cyclase family & $2 \mathrm{E}-28$ & & & 0.81 & & 0.67 & 0.81 \\
\hline
\end{tabular}

Transcription

CB329931

DV035417

CB330050

CB330261

CB330874

Energy

DV035668:

CB330656

CB330553

CB329932

CB330898

CB330249

CB330265

CB330941

Subcellular localization

DV037573i

tubulin alpha-6 chain (TUA6)

ATPase alpha subunit

chlorophyll A-B binding protein /

LHCI type I (CAB)

chlorophyll A-B binding protein /

LHCI type III (LHCA3.1)

chlorophyll A-B binding protein /

LHCII type I (LHB1B2)

chlorophyll A-B binding protein /

LHCII type II (LHCB2.2)

chlorophyll A-B binding protein /

LHCII type III (LHCB3)

cytochrome B6-F complex iron-

sulfur subunit, chloroplast / Rieske

iron-sulfur protein / plastoquinol-

plastocyanin reductase (petC)

cytochrome $\mathrm{c}$ oxidase subunit $\mathrm{Vc}$

family protein / COX5C family

protein

Transport

CB330313

DV037387'

CB330259

CB330095 acyl carrier family protein / ACP

family protein

heat shock protein 70 (HSP70-1)

ferredoxin, chloroplast (PETF)

lipid transfer protein 3 (LTP3)
1E-63

$1 \mathrm{E}-108$

$1 \mathrm{E}-127$

6E-99

1E-137

$1 \mathrm{E}-123$

$6 \mathrm{E}-84$

4E-22

$5 \mathrm{E}-52$

$1 \mathrm{E}-44$

0.70

0.80

0.95

0.68

1.29

0.67

0.66

0.54

0.50

$1 \mathrm{E}-117$

2E-33

$7 \mathrm{E}-20$

0.50

-0.51
0.66
-0.80

0.59
0.42

0.89

50.50

0.55

0.81

0.63

$0.66 \quad 0.66$

$0.71 \quad 0.62$

$0.84 \quad 0.85$

$0.63 \quad 0.54$

$0.94 \quad 0.79$

$0.64 \quad 0.64$

$0.71 \quad 0.67$

$\begin{array}{ll}-0.50 & -0.45\end{array}$

$\begin{array}{ll}0.75 & 0.67\end{array}$

$\begin{array}{ll}-0.82 & -1.09\end{array}$

continued next page 
Table 1. Continued.

\begin{tabular}{|c|c|c|c|c|c|c|c|c|}
\hline \multirow{2}{*}{$\begin{array}{l}\text { GenBank } \\
\text { accession no. }\end{array}$} & \multirow[b]{2}{*}{ Gene description } & \multirow[b]{2}{*}{$\begin{array}{c}\text { E- } \\
\text { value }^{z}\end{array}$} & \multicolumn{6}{|c|}{ M-values ${ }^{\mathrm{y}}$} \\
\hline & & & $\begin{array}{l}\text { VT }^{\mathrm{x}}- \\
\text { SPFMV }\end{array}$ & $\begin{array}{l}\mathrm{VT}^{\mathrm{x}}- \\
\mathrm{SPCSV}\end{array}$ & $\begin{array}{l}V^{\mathrm{x}}- \\
\text { SPVD }\end{array}$ & $\begin{array}{l}\text { SPFMV } \\
\text {-SPCSV }\end{array}$ & $\begin{array}{r}\text { SPFMV } \\
\text {-SPVD }\end{array}$ & $\begin{array}{l}\text { SPCSV } \\
\text {-SPVD } \\
\end{array}$ \\
\hline CB330457 & $\begin{array}{l}\text { lipid transfer protein (LTP) family } \\
\text { protein }\end{array}$ & $3 \mathrm{E}-28$ & & 0.48 & & & & \\
\hline \multicolumn{9}{|c|}{ Interaction with the environment } \\
\hline DV034646 & $\begin{array}{l}\text { gibberellin-regulated protein } 5 \\
\text { (GASA5) }\end{array}$ & $3 \mathrm{E}-33$ & & & -0.57 & & -0.61 & -0.54 \\
\hline \multicolumn{9}{|c|}{ Signal transduction } \\
\hline CB330823 & $\begin{array}{l}\text { mitogen-activated protein kinase / } \\
\text { MAPK, putative }\end{array}$ & $5 \mathrm{E}-70$ & & & -1.31 & & -1.42 & \\
\hline DV035511 & receptor-like protein kinase, putative & $2 \mathrm{E}-19$ & & & -0.60 & & -0.64 & -0.58 \\
\hline \multicolumn{9}{|c|}{ Unclassified proteins } \\
\hline DV035493 & $\begin{array}{l}\text { 26S proteasome regulatory subunit } \\
\text { S2 (RPN1) }\end{array}$ & $8 \mathrm{E}-08$ & -0.51 & -0.78 & -0.40 & & & \\
\hline DV036499 & ankyrin repeat family protein & $1 \mathrm{E}-63$ & & & 0.44 & & & 0.43 \\
\hline CB329954 & $\mathrm{BTB} / \mathrm{POZ}$ domain-containing protein & $4 \mathrm{E}-56$ & & & 0.51 & & 0.46 & \\
\hline DV035142 & $\begin{array}{l}\text { chloroplast nucleoid DNA-binding } \\
\text { protein-related }\end{array}$ & 7E-39 & & & -0.58 & & -0.55 & -0.61 \\
\hline CB330921 & $\begin{array}{l}\text { dehydration-induced protein } \\
\text { (ERD15) }\end{array}$ & $1 \mathrm{E}-31$ & & & -0.45 & & -0.52 & \\
\hline CB330447 & $\begin{array}{l}\text { DNAJ heat shock N-terminal } \\
\text { domain-containing protein }\end{array}$ & $2 \mathrm{E}-21$ & & & & -0.83 & & -0.66 \\
\hline DV036723 & $\begin{array}{l}\text { dormancy-associated protein, } \\
\text { putative (DRM1) }\end{array}$ & $1 \mathrm{E}-30$ & & & -0.55 & & -0.55 & \\
\hline CB330810 & emp24/gp25L/p24 protein-related & $1 \mathrm{E}-99$ & & & 0.59 & & & \\
\hline DV037327 & epoxide hydrolase, putative & $1 \mathrm{E}-27$ & & -0.49 & - & -0.43 & & \\
\hline CB330021 & fructosamine kinase family protein & $1 \mathrm{E}-144$ & & & 0.62 & & & \\
\hline CB330841 & Ferredoxin I, chloroplast precursor & $5 \mathrm{E}-33$ & & & -0.63 & & -0.65 & -0.58 \\
\hline CB330388 & hevein-like protein (HEL) & 9E-48 & & & 0.56 & & & \\
\hline DV035503 & $\begin{array}{l}\text { methyltransferase MT-A70 family } \\
\text { protein }\end{array}$ & $6 \mathrm{E}-57$ & & -0.66 & -0.45 & & & \\
\hline DV036783 & myb family transcription factor & $5 \mathrm{E}-25$ & & & & & & -0.52 \\
\hline DV035732 & $\begin{array}{l}\text { pentatricopeptide (PPR) repeat- } \\
\text { containing protein }\end{array}$ & $1 \mathrm{E}-79$ & & & -0.40 & & -0.42 & -0.35 \\
\hline CB330263 & photosystem II $10 \mathrm{kDa}$ polypeptide & $3 \mathrm{E}-42$ & & 0.76 & 0.97 & & 0.97 & 0.63 \\
\hline CB330912 & $\begin{array}{l}\text { photosystem II core complex } \\
\text { proteins psbY, chloroplast (PSBY) / } \\
\text { L-arginine metabolising enzyme }\end{array}$ & $1 \mathrm{E}-29$ & & & 1.13 & & 0.87 & 0.90 \\
\hline CB330154 & plastocyanin & $1 \mathrm{E}-51$ & 0.50 & 0.55 & 0.83 & & & \\
\hline DV037560 & $\begin{array}{l}\text { polygalacturonase inhibiting protein } \\
2 \text { (PGIP2) }\end{array}$ & $2 \mathrm{E}-55$ & & & -0.38 & & -0.42 & -0.34 \\
\hline DV036718 & Reticulon family protein (RTNLB3) & $3 \mathrm{E}-61$ & & & -0.74 & & -0.71 & -0.68 \\
\hline DV034984 & Riboflavin synthase & $3 \mathrm{E}-28$ & & & -0.37 & & & \\
\hline CB330073 & senescence-associated family protein & $3 \mathrm{E}-61$ & & & -0.70 & & -0.73 & -0.75 \\
\hline DV037482 & $\begin{array}{l}\text { Sporamin (Kunitz type trypsin } \\
\text { inhibitor family) }\end{array}$ & 0.0 & & -0.72 & & -0.79 & & 0.65 \\
\hline CB330386 & $\begin{array}{l}\text { SWIB complex BAF } 60 \mathrm{~b} \text { domain- } \\
\text { containing protein }\end{array}$ & $8 \mathrm{E}-33$ & & & 0.71 & & 0.65 & 0.51 \\
\hline DV037510 & $\begin{array}{l}\text { TATA-binding protein-associated } \\
\text { factor TAFII } 55 \text { family protein }\end{array}$ & $3 \mathrm{E}-58$ & & & -0.53 & & -0.54 & -0.44 \\
\hline CB330112 & wound-responsive family protein & $3 \mathrm{E}-24$ & & & -0.67 & & -0.46 & -0.44 \\
\hline DV034644 & yippee family protein & $1 \mathrm{E}-17$ & & & -0.81 & & -0.46 & -0.44 \\
\hline DV035849 & $\begin{array}{l}\text { zinc finger ( } \mathrm{C} 2 \mathrm{H} 2 \text { type }) \text { family } \\
\text { protein }\end{array}$ & 2E-99 & & -0.50 & & & & \\
\hline
\end{tabular}

Expectation value; the lower the E-value, the more significant is the score.

yM-values $=\log 2$ (fold change)

xpositive and negative M-values denote higher and lower expression levels in VT treatment, respectively 
Table 2. Quantitative real-time polymerase chain reaction (Q-RT-PCR) primers used for validation of sweetpotato microarray results. The primers were designed using Primer Express (version 2.0; Applied Biosystems, Foster City, Calif.).

\begin{tabular}{|c|c|c|c|}
\hline Gene & $\begin{array}{c}\text { GenBank } \\
\text { accession no. }\end{array}$ & $\begin{array}{c}\text { Primer } \\
\text { name }\end{array}$ & Sequence $5^{\prime}-3^{\prime}$ \\
\hline \multirow[t]{2}{*}{ Cat2 } & DV036659 & Fwd & GGGCCAATTCTGTTGGAAGA \\
\hline & & Rev & TCTGGGATCCTTTCACGAGTG \\
\hline \multirow[t]{2}{*}{ ERD15 } & CB330921 & Fwd & CCAGCAGCAGGGAACAGAAT \\
\hline & & Rev & CATCGAGATCAATGGTATCAGGC \\
\hline \multirow[t]{2}{*}{ TIR-NBS-LRR } & DV036322 & Fwd & TCACCTCTTTGCAGCGTTGT \\
\hline & & Rev & GTCCTTTACGGAGCTCTTCTTCAT \\
\hline \multirow[t]{2}{*}{ HSP70-1 } & DV037387 & Fwd & CTTGGTCTTGAAACTGCCGG \\
\hline & & Rev & TTCTTGGTGGGAATGGTGGT \\
\hline \multirow[t]{2}{*}{ LHCB3 } & CB330249 & Fwd & TTTTCTGCCCAAACTCCTTCAT \\
\hline & & Rev & AAACCAGCAGTGTCCCATCC \\
\hline \multirow[t]{2}{*}{ Ankyrin } & DV036499 & Fwd & CATGTCCACCATGCTTGAGAGT \\
\hline & & Rev & TGCGTGCCATTCGTTCTTC \\
\hline \multirow[t]{2}{*}{ MT2A } & CB330120 & Fwd & CGGGTGCAAGATGTACCCAG \\
\hline & & Rev & CGCCAAGAACAAGGGTCTCA \\
\hline
\end{tabular}

Table 3. Comparison of average fold-change values between quantitative real-time polymerase chain reaction (Q-RT-PCR) and microarray assays of randomly selected genes differentially expressed in sweet potato virus disease (SPVD)-affected sweetpotato plants compared to virus-tested controls. Positive fold changes denote down-regulation, while negative values represent induction in SPVD affected plants. All fold changes were statistically significant using a $P$ value cutoff of 0.05 (after Holm multiple testing correction for microarray data).

\begin{tabular}{lccc}
\hline & \multirow{2}{*}{$\begin{array}{c}\text { GenBank } \\
\text { accession } \\
\text { nene name }\end{array}$} & \multicolumn{2}{c}{ Fold change } \\
\cline { 3 - 4 } catalase 2 & DV036659 & -1.47 & -1.54 \\
dehydration-induced protein (ERD15) & CB330921 & -2.13 & -1.37 \\
disease resistance protein (TIR-NBS-LRR) & DV036322 & -2.35 & -1.85 \\
heat shock protein 70 (HSP70-1) & DV037387 & -1.73 & -1.42 \\
chlorophyll A-B binding protein/LHCII & CB330249 & 3.74 & 1.59 \\
type III (LHCB3) & & & \\
ankyrin repeat family protein & DV036499 & 1.38 & 1.36 \\
metallothionein protein, putative (MT2A) & CB330120 & 1.40 & 1.35 \\
\hline
\end{tabular}

Another group of gene products implicated in the responses of plants to pathogens and other stresses are peroxidases (Lagrimini and Rothstein, 1987; Yan et al., 2002). Peroxidases have been shown to be involved in scavenging of $\mathrm{H}_{2} \mathrm{O}_{2}$ from peroxisomes (Wang et al., 1999). The down-regulation of a peroxidase gene (CB330630) in SPVDaffected plants that may be associated with the prevention of downstream activation of ROS-dependent host defense responses, suggests that the differential expression of this gene is directed by the two interacting viruses.

Many of the pathogen-related (PR) proteins exhibit enzymatic activities. A major group of such pathogenesis related proteins, reported from tomato plants (Lycopersicon esculentum Mill.), are proteases. These proteases are involved in specific proteolytic events in the extracellular matrix during infection. (Tornero et al., 1997; Vera and Conejero, 1988). A member of this group (PR-P69), which was later identified as subtilisin-like proteases (Tornero et al., 1996), was induced in plants infected with citrus exocortis viroid (Vera and Conejero, 1988). In this experiment, a subtilase gene (CB330070) was down-regulated only in SPVD affected plants. This and other down-regulated PR genes reported in this study (Table 1) may play an important role in this host's defense mechanism since their transcriptional suppression, caused only by the interacting viruses, may be linked to the severe disease development observed in SPVD-affected plants.

was accompanied by increased levels of ROS such as $\mathrm{H}_{2} \mathrm{O}_{2}$. The down regulation of the ankyrin gene in SPVD affected plants may be indirectly associated with the up-regulation of some of the other stress response genes, reported in this study through the activity of ROS, or the gene may simply be repressed by the virus. However, in some cases excessive amounts of these toxic compounds interfere with the efficiency of the host to restrict pathogen infection (Moreno et al., 2005).

A particularly interesting gene that is up-regulated in SPVD compared to all other treatments is eukaryotic translation initiation factor 5A(eIF-5A) (DV037506). This protein factor contains the unique amino acid, hypusine. In $A$. thaliana there are three isoforms of eIF-5A, two of which are involved in senescence and the other one in cell division (Gatsukovich, 2004; Thompson et al., 2004). Transgenic $A$. thaliana plants with decreased deoxyhypusine synthase (DHS) levels, the enzyme that is required for eIF5A activation, showed increased resistance to lethal drought stress (Wang et al., 2003). In humans it is a crucial co-factor of the Rev pathway (Hoffman et al., 2001) essential for HIV1 replication (Pollard and Malim, 1998). Suppression of DHS has been suggested as a mechanism for antiretroviral therapies (Hauber et al., 2005). The up-regulation of eIF-5A in SPVD is most likely related to leaf senescence. However the possibility that eIF-5A has an additional role in virus replication (as in humans) cannot be excluded.
Epoxide hydrolase (DV037327), induced in SPCSV plants, is also induced in tobacco (Nicotiana tabacum L.) leaves infected with TMV (Guo et al., 1998). Catalase II (DV036659), an enzyme that breaks down $\mathrm{H}_{2} \mathrm{O}_{2}$ and is inhibited by salicylic acid (Conrath et al., 1995), is induced in SPVD affected plants. In tomato plants infected with cucumber mosaic virus (CMV) and D satellite RNA, the induction of catalase II was associated with accumulation of $\mathrm{H}_{2} \mathrm{O}_{2}$ (Xu et al., 2003). ERD15 (CB330921), a gene that has been shown to be induced by the addition of external $\mathrm{H}_{2} \mathrm{O}_{2}$ in A. thaliana (Dunaeva and Adamska, 2001), was also up-regulated in SPVD (Kiyosue et al., 1994), but was also induced in A. thaliana plants inoculated with plant-growth-promoting rhizobacteria (PGPR) (Timmusk and Wagner, 1999). These plants were more resistant to Erwinia carotovora (Jones) Bergey et al. Timmusk and Wagner (1999) speculated that the unexpected induction of ERD15 was a result of stunting of roots of inoculated plants. Our results suggest a probable role for ERD15 in general stress response.

The induction of polyubiquitin (CB330102) and heat-shock protein 70 (HSP70) (DV037387) during virus infections have been reported earlier (Aranda et al., 1996; Escaler et al., 2000; Whitham et al., 2003). Glotzer et al. (2000) reported that induction of HSP70 and HSP40 promote adenovirus infection. Our results indicate that HSP70 was induced in SPVD compared to all other treatments. It is unclear whether this indicates nontransient plants. ERD15 was first identified as a drought responsive gene 
accumulation of HSP70, or is due to new cells continuously inducing HSP70 transiently as they become infected (Whitham et al. 2003). It should be noted that HSP70 was not induced in SPCSV. Like other members of Closteroviridae, SPCSV encodes its own HSP70 homolog (Kreuze et al., 2002) that assists with movement through the plasmodesmata (Prokhnevsky et al., 2002). Aparicio et al. (2005) recently showed that induction of HSP70 is a general response to protein accumulation in the cytosol. The induction of HSP70 in SPVD may be due to protein accumulation associated with increased levels of SPFMV during the dual infection. The function of HSP70 for virus families, other than Closteroviridae has not been proven, but a similar role in cell-to-cell trafficking seems likely (Aoki et al., 2002; Aparicio et al., 2005).

The induction of host PR genes during the course of a severe disease, as in SPVD-affected plants, has also been reported by $\mathrm{Xu}$ et al. (2003). The induction of multiple defense responses in tomato plants infected with CMV and D satellite RNA were insufficient in conferring any form of resistance resulting even in plant death. Since infection without the D satellite RNA does not lead to any severe outcome, it appears that these two phenomena, dual infection with SPCSV and SPFMV, and CSV and D satellite RNA, may trigger similar responses in the host.

A caveat to the present study is that the genes on the array represent only a small proportion of the total sweetpotato genome. Many genes that may be differentially expressed are not detected in this study. Some of these may be critical in understanding host-pathogen relationships and the underlying factors that promote the synergistic response in sweetpotato.

Since SPVD and its viral components were first described by Schaefers and Terry (1976), several hypotheses on the mechanism underlying this disease have been formulated (Kreuze, 2002). One suggests that SPCSV suppresses the resistance mechanism in the host, leading to enhanced multiplication of SPFMV. Another proposed mechanism involves a form of interaction between the two viruses (HC-Pro of SPFMV and P-Pro of SPCSV) leading to enhancement of SPFMV. It was further hypothesized that the symptoms of SPVD were induced primarily as a result of the enhanced replication of SPFMV.

It is known that SPCSV, a phloem-limited virus, does not exit the phloem even when coinfecting with SPFMV (Karyeija et al., 2000). Furthermore, SPCS V, whose titers are significantly greater than those of SPFMV in single infections, remains relatively unchanged or is reduced during SPVD (Karyeija et al., 2000; Kokkinos, 2006). Kokkinos (2006) and Mukasa (2004) showed that SPCSV enhances replication of several potyviruses in sweetpotato. However, SPFMV-C does not interact with SPCSV to cause the same SPVD symptoms even though its titer is similarly enhanced as that of SPFMV-RC (Kokkinos, 2006). This suggests that enhancement of potyvirus replication by itself is not sufficient to induce the severe symptoms associated by SPVD. Kreuze et al. (2005) recently described two proteins, RNase III and p22, of SPCSV that suppress RNA silencing in Nicotiana benthamiana Domin. Interestingly, agronaute 1 (AGO1), a gene involved in RNA silencing (Okamura et al., 2004), was not differentially expressed among any of the treatments in the present study (data not shown). It has been reported that AGO1 mutants in A. thaliana are impaired in virus resistance (Morel et al., 2002), but a recent study showed that AGO1 does not recruit virus-derived siRNAs (Baumberger and Baulcombe, 2005). Since the mechanism by which SPCSV RNase III and p22 suppress RNA silencing remains to be elucidated, it is difficult to speculate on how the expression of host RNA silencing-related genes would be affected. Finally, the present study did not show any clear indication why certain defense related genes where up regulated and some were down regulated or why so many more genes were differentially expressed in the duel infections.

Future host gene expression studies should include other virus combinations, including SPFMV-C, as well as resistant cultivars if they are available. In addition, the study should be broadened to include several time points after infection and an array where more sweetpotato genes are represented.

\section{Literature Cited}

Aarts, N., M. Metz, E. Holub, B.J. Staskawicz, M.J. Daniels, and J.E. Parker. 1998. Different requirements for EDS1 and NDR1 by disease resistance genes define at least two $R$ gene-mediated signaling pathways in Arabidopsis. Proc. Natl. Acad. Sci. USA 95:10306-10311.

Aoki, K., F. Kragler, B. Xoconostle-Cázares, and W.J. Lucas. 2002. A subclass of plant heat shock cognate 70 chaperones carries a motif that facilitates trafficking through plasmodesmata. Proc. Natl. Acad. Sci. USA 99:16342-16347.

Aparicio, F., C.L. Thomas, C. Lederer, Y. Niu, D. Wang, and A.J. Maule. 2005. Virus induction of heat shock protein 70 reflects a general response to protein accumulation in the plant cytosol. Plant Physiol. 138:529-536.

Aranda, M.A., M. Escaler, D. Wang, and A.J. Maule. 1996. Induction of HSP70 and polyubiquitin expression associated with plant virus replication. Proc. Natl. Acad. Sci. USA 93:15289-15293.

Baumberger, N. and D.C. Baulcombe. 2005.Arabidopsis ARGONAUTE1 is an RNA slicer that selectively recruits microRNAs and short interfering RNAs. Proc. Natl. Acad. Sci. USA 102:11928-11933.

Brunt, A.A., K. Crabtree, M.J. Dallwitz, A.J. Gibbs, and L. Watson. 1996. Viruses of plants. Descriptions and lists from the VIDE database. CABI, Wallingford, England.

Clark, C.A. and M.W. Hoy. 2006. Effects of common viruses on yield and quality of Beauregard sweetpotato in Louisiana. Plant Dis. 90:83-88.

Conrath, U., Z. Chen, J.R. Ricigliano, and D.F. Klessig. 1995. Two inducers of plant defense responses, 2,6-dichloroisonicotinic acid and salicylic acid, inhibit catalase activity in tobacco. Proc. Natl. Acad. Sci. USA 92:7143-7147.

De Vos, M., V.R. Van Oosten, R.P.M. Van Poecke, J.A. Van Pelt, M.J. Pozo, M.J. Mueller, A.J. Buchala, J-P. Métraux, L.C. Van Loon, M. Dicke, and C.M.J. Pieterse. 2005. Signal signature and transcriptome changes of Arabidopsis during pathogen and insect attack. Mol. Plant Microbe Interactions 18:923-937.

Di Feo, L., S.F. Nome, E. Biderbost, S. Fuentes, and L. Salazar. 2000. Etiology of sweet potato chlorotic dwarf disease in Argentina. Plant Dis. 84:35-39.

Dowd, C., I.W. Wilson, and H. McFadden. 2004. Gene expression profile changes in cotton root and hypocotyls tissues in response to infection with Fusarium oxysporum f. sp. vasinfectum. Mol. Plant Microbe Interactions 17:654-667.

Dudoit, S., Y.H. Yang, M.J. Callow, and T.P. Speed. 2002. Statistical methods for identifying differentially expressed genes in replicated cDNA microarray experiments. Statistica Sinica 12:111-139.

Dunaeva, M. and I. Adamska. 2001. Identification of genes expressed in response to light stress in leaves of Arabidopsis thaliana using RNA differential display. European J. Biochem. 268:5521-5529.

Escaler, M., M.A. Aranda, C.L. Thomas, and A.J. Maule. 2000. Pea embryonic tissues show common responses to the replication of a wide range of viruses. Virology 267:318-325.

Food and Agriculture Organization of the United Nations. FAOSTAT data, 2005. 21 Feb. 2006. <http://faostat.fao.org/faostat/form?collection= Production.Crops.Primary \&Domain=Production \&servlet $=1 \&$ hasbul $\mathrm{k}=0$ \&version $=$ ext\&language $=\mathrm{EN}>$.

Gatsukovich, Y.2004. Characterization of eukaryotic translation initiation factor 5A-2 (eIF5A-2) in Arabidopsis thaliana: Effects of wounding and pathogen attack. MS Thesis, Univ. of Waterloo, Waterloo, Ont., Can. 
Gibly, A., A. Bonshtien, V. Balaji, P. Debbie, G. B. Martin, and G. Sessa. 2004. Identification and expression profiling of tomato genes differentially regulated during a resistance response to Xanthomonas campestris pv. vesicatoria. Mol. Plant Microbe Interactions 17:1212-1222.

Gibson, R.W., I. Mpembe, T. Alicai, E.E. Carey, R.O.M. Mwanga, S.E. Seal, and H.J. Vetten. 1998. Symptoms, aetiology and serological analysis of sweet potato virus disease in Uganda. Plant Pathol. 47:95-102.

Glazebrook, J. 2001. Genes controlling expression of defense responses in Arabidopsis - 2001 status. Curr. Opin. Plant Biol. 4:301-308.

Glotzer, J.B., M. Saltik, S. Chiocca, A.I. Michou, P. Moseley, and M. Cotten. 2000. Activation of heat-shock response by an adenovirus is essential for virus replication. Nature 407:207-211.

Golem, S. and N.J. Culver. 2003. Tobacco mosaic virus induced alterations in the gene expression profile of Arabidopsis thaliana. Mol. Plant Microbe Interactions 16:681-688.

Guo, A., J. Durner, and D.F. Klessig. 1998. Characterization of a tobacco epoxide hydrolase gene induced during the resistance response to TMV. Plant J. 15:647-656.

Gutiérrez, D.L., S. Fuentes, and L.F. Salazar. 2003. Sweetpotato virus disease (SPVD): Distribution, incidence, and effect on sweetpotato yield in Peru. Plant Dis. 87:297-302.

Hahn, S.K. 1976. Effects of viruses (SPVD) on growth and yield of sweet potato. Expt. Agr. 15: 253-256.

Hauber, I., D. Bevec, J. Heukeshoven, F. Krätzer, F. Horn, A. Choidas, T. Harrer, and J. Hauber. 2005. Identification of cellular deoxyhypusine synthase as a novel target for antiretroviral therapy. J. Clinical Investigation 115:76-85.

Hofmann, W., B. Reichart, A. Ewald, E. Müller, I. Schmitt, R.H. Stauber, F. Lottspeich, G.M. Jockusch, U. Scheer, J. Hauber, and M-C. Dabauvalle. 2001. Cofactor requirements for nuclear export of Rev response element (RRE)- and constitutive transport element (CTE)containing retroviral RNAs. An unexpected role for actin. J. Cell Biol. 152:895-910.

Holm, S. 1979. A simple sequentially rejective Bonferroni test procedure. Scandinavian J. Stat. 6:65-70.

Hull, R. 2002. Matthews' plant virology. 4th ed. Academic, San Diego, Calif.

IsHak, J.A., J.F. Kreuze, A. Johansson, S.B. Mukasa, F. Tairo, F.M. Abo El-Abbas, and J.P. Valkonen. 2003. Some molecular characteristics of three viruses from SPVD-affected sweet potato plants in Egypt. Arch. Virol. 148:2449-2460.

Izaguirre-Mayoral, M.L., R.C. de Uzcategui, and O. Carballo. 1993. Crassulacean acid metabolism in two species of orchids infected by tobacco mosaic virus-orchid strain and/or cymbidium mosaic virus. J. Phytopathol. 137:272-282.

Jian, A.N., T.A. Tokuyasu, A.M. Snijders, R. Segraves, D.G. Albertson, and D. Pinkel. 2002. Fully automatic quantification of microarray data. Genome Res. 12:325-332.

Karyeija, R.F., R.W. Gibson, and J.P.T. Valkonen. 1998a. The significance of sweet potato feathery mottle virus in subsistence sweetpotato production in Africa. Plant Dis. 82:4-15.

Karyeija, R.F., R.W. Gibson, and J.P.T. Valkonen. 1998b. Resistance to sweet potato virus disease (SPVD) in wild east African Ipomoea. Ann. Appl. Biol. 133:39-44.

Karyeija, R.F., J.F. Kreuze, R.W. Gibson, and J.P.T. Valkonen. 2000. Synergistic interactions of a potyvirus and a phloem-limited crinivirus in sweetpotato plants. Virology 269:26-36.

Kato-Maeda, M., Q. Gao, and P.M. Small. 2001. Microarray analysis of pathogens and their interaction with hosts. Cellular Microbiol. 3:713-719.

Kiyosue, T., K. Yamaguchi-Shinozaki, and K. Shinozaki. 1994. Cloning of cDNAs for genes that are early-responsive to dehydration stress (ERDs) in Arabidopsis thaliana L.: Identification of three ERDs as HSP cognate genes. Plant Mol. Biol. 25:791-798.

Kokkinos, C.D. 2006. Assessment of interactions among viruses infecting sweetpotato. PhD Diss., Louisiana State Univ., Baton Rouge.
Kokkinos, C.D. and C.A. Clark. 2004. Assessment of interactions among important sweetpotato viruses using real-time quantitative PCR. Phytopathology 94:S54. (Abstr.).

Kokkinos, C.D. and C.A. Clark. 2006. Real-time PCR assays for detection and quantification of sweetpotato viruses. Plant Dis. (In press).

Kreuze, J. 2002. Molecular studies on the sweetpotato virus disease and its two causal agents. Doctoral Thesis, Swedish University of Agricultural Sciences, Uppsala, Sweden.

Kreuze, J.F., E.I. Savenkov, and J.P.T. Valkonen. 2002. Complete genome sequence and analyses of the subgenomic RNAs of sweet potato chlorotic stunt virus reveal several new features for the genus Crinivirus. J. Virol. 76:9260-9270.

Kreuze, J.F., E.I. Savenkov, W.Cuellar, X. Li, and J.P.T. Valkonen. 2005. Viral class 1 RNase III involved in suppression of RNA silencing. J. Virol. 79:7227-7238.

Largimini, L.M. and S. Rothstein. 1987. Tissue specificity of tobacco peroxidase isozymes and their induction by wounding and tobacco mosaic virus infection. Plant Physiol. 84:438-442.

Loebenstein, G. and I. Harpaz. 1960. Virus diseases of sweetpotatoes in Israel. Phytopathology 50:100-104.

Moissiard, G. and O. Voinnet. 2004. Viral suppression of RNA silencing in plants. Mol. Plant Pathol. 5:71-82.

Morel, J.B., C. Godon, P. Mourrain, C. Béclin, S. Boutet, F. Feuerbach, F. Proux, and H. Vaucheret. 2002. Fertile hypomorphic ARGONAUTE (ago1) mutants impaired in post-transcriptional gene silencing and virus resistance. Plant Cell 14:629-639.

Moreno, J.I., R. Martín, and C. Castresana. 2005. Arabidopsis SHMT1, a serine hydroxymethyltransferase that functions in the photorespiratory pathway influences resistance to biotic and abiotic stress. Plant J. 41:451-463.

Moy, P., D. Qutob, B.P. Chapman, I. Atkinson, and M. Gijzen. 2004. Patterns of gene expression upon infection of soybean plants by $P$ hytophthora sojae. Mol. Plant Microbe Interactions 17:1051-1062.

Mukasa, S. 2004. Genetic variability and interactions of three sweetpotato infecting viruses. PhD Diss., Swedish Univ. of Agr. Sci., Uppsala, Sweden.

Naidu, R.A., M. Krishnan, M.V. Nayadu, and A. Gnanam. 1986. Studies on peanut green mosaic virus infected peanut (Arachis hypogaea L.) leaves: III Changes in the polypeptides of photosystem II particles. Physiol. Mol. Plant Pathol. 29:53-58.

Ngeve, J.M. 1990. Yield stability and yield depression in sweet potato cultivars susceptible to the sweet potato virus disease. J. Hort. Sci. 65:225-230.

Okamura, K., A. Ishizuka, H. Siomi, and M.C. Siomi. 2004. Distinct roles for Argonaute proteins in small RNA-directed RNA cleavage pathways. Genes Dev. 18:1655-1666.

Pio-Ribeiro, G., S. Winter, R.L. Jarret, J.W. Demski, and R.I. Hamilton. 1996. Detection of sweet potato virus disease-associated closterovirus in a sweetpotato accession in the United States. Plant Dis. 80:551-554.

Pollard, V.W. and M.H. Malim. 1998. The HIV-1 Rev protein. Ann. Rev. Microbiol. 52:491-532.

Prokhnevsky, A.I., V.V. Peremyslov, A.J. Napuli, and V.V. Dolja. 2002. Interaction between long-distance transport factor and Hsp70-related movement protein of beet yellows virus. J. Virol. 76:11003-11011.

Rosa, G.J.M., J.P. Steibel, and R.J. Tempelman. 2005. Reassessing design and analysis of two-colour microarray experiments using mixed effects models. Comparative Functional Genomics 6:123-131.

Salazar, L.E. and S. Fuentes. 2001. Current knowledge on major virus diseases of sweetpotatoes. Proc. Intl. Workshop on Sweetpotato Cultivar Decline Study. 8-9 Sept. 2000, Miyakonojo, Jpn.

Schaefers, G.A. andE.R. Terry. 1976. Insect transmission of sweet potato disease agents in Nigeria. Phytopathology 66:642-645.

Schena, M., D. Shalon, R.W. Davis, and P.O. Brown. 1995. Quantitative monitoring of gene expression patterns with a complementary DNA microarray. Science 270:467-470.

Schoof, H., P. Zaccaria, H. Gundlach, K. Lemcke, S. Rudd, G. Kolesov, R. Arnold, H.W. Mewes. and K.F. Mayer. 2002. MIPS Arabidopsis 
thaliana Database (MAtDB): An integrated biological knowledge resource based on the first complete plant genome. Nucleic Acids Res. 30:91-93.

Smyth, G.K. and T.P. Speed. 2003. Normalization of cDNA microarray data. Methods 31:265-273.

Smyth, G.K. 2004. Linear models and empirical Bayes methods for assessing differential expression in microarray experiments. Stat. Applications Genet. Mol. Biol. 3(1):article 3. 7 Mar. 2006. <http://www. bepress.com/sagmb/vol3/iss1/art3/>.

Souto, E.R, J. Sim, J. Chen, R. A. Valverde, and C. A. Clark. 2003. Properties of strains of sweet potato feathery mottle virus and two newly recognized potyviruses infecting sweet potato in the United States. Plant Dis. 87:1226-1232.

Tairo, F., S.B. Mukasa, R.A.C. Jones, A. Kullaya, P.R. Rubaihayo, and J.P.T. Valkonen. 2005. Unravelling the genetic diversity of the three main viruses involved in sweet potato virus disease (SPVD) and its implications. Mol. Plant Pathol. 6:199-211.

Thompson, J.E., M.T. Hopkins, C. Taylor, and T-W. Wang. 2004. Regulation of senescence by eukaryotic translation initiation factor 5A: implications for plant growth and development. Trends Plant Sci. 9:174-179.

Timmusk, S. and E.G.H. Wagner. 1999. The plant-growth-promoting rhizobacterium Paenibacillus polymyxa induces changes in Arabidopsis thaliana gene expression: A possible connection between biotic and abiotic stress responses. Mol. Plant Microbe Interactions 12:951-959.

Tornero, P., V. Conejero, and P. Vera. 1996. Primary structure and expression of a pathogen-induced protease (PR-P69) in tomato plants: Similarity of functional domains to subtilisin-like endoproteases. Proc. Natl. Acad. Sci. USA 93:6332-6337.

Tornero, P., V. Conejero, and P. Vera. 1997. Identification of a new pathogen-induced member of the subtilisin-like processing protease family from plants. J. Biol. Chem. 272:14412-14419.

Valverde, R.A., A.G. Lozano, J. Navas-Castillo, A. Ramos, and F. Valdez. 2004. First report of sweetpotato chlorotic stunt virus and sweetpotato feathery mottle virus infecting sweet potato in Spain. Plant Dis. 88:428. van Kooten, O., C. Meurs, and L.C. van Loon. 1990. Photosynthetic electron transport in tobaccoleaves infected with tobacco mosaic virus. Physiol. Plant. 80:446-542.

Vera, P. and V. Conejero. 1988. Pathogenesis-related proteins of tomato. P-69 as an alkaline endoproteinase. Plant Physiol. 87:58-63.

Voinnet, O. 2005 Induction and suppression of RNA silencing: Insights from viral infections. Nature Rev. Genet. 6:206-221.

Wang, J., H.Zhang, and R.D. Allen. 1999. Overexpression of an arabidopsis putative peroxisomal ascorbate peroxidase gene in tobacco increases protection against oxidative stress. Plant Cell Physiol. 40:725-732.

Wang, T-W., L. Lu, C-G., Zhang, C. Taylor, and J.E. Thompson. 2003. Pleiotropic effects of suppressing deoxyhypusine synthase expression in Arabidopsis thaliana. Plant Mol. Biol. 52:1223-1235.

Wettenhall, J.M. and G.K. Smyth. 2004 limmaGUI: A graphical user interface for linear modeling of microarray data. Bioinformatics 1:1-2.

Whitham, S.A., S. Quan, H-R. Chang, B. Cooper, B. Estes, T. Zhu, X. Wang, and Y-M. Hou. 2003. Diverse RNA viruses elicit the expression of common sets of genes in susceptible Arabidopsis thaliana plants. Plant J. 33:271-283.

Xu, P., E.B. Blancaflor, and M.J. Roossinck. 2003. In spite of induced multiple defense responses, tomato plants infected with cucumber mosaic virus and D satellite RNA succumb to systemic necrosis. Mol. Plant Microbe Interactions 16:467-476.

Yan, J., J. Wang, and H. Zhang. 2002. An ankyrin repeat-containing protein plays a role in both disease and antioxidation metabolism. Plant J. 29:193-202.

Yun W.S., Y.H. Lee, and K.H. Kim. 2002. First report of sweet potato latent virus and sweet potato chlorotic stunt virus isolated from sweet potato in Korea. Plant Pathol. J. 18:126-129.

Zhang L-M,, Q-M. Wang, D-F. Ma, and Y. Wang. 2005. Major viruses and effect of major virus diseases and virus-eliminating meristem culture on sweetpotato yield and quality in China. Acta Botanica Boreali-Occidentalia Sinica 25:316-320.

Zhu, H., C.P. Cong, G. Mamtora, T. Gingeras, and T. Shenk. 1998. Cellular gene expression altered by human cytomegalovirus: Global monitoring with oligonucleotide arrays. Proc. Natl. Acad. Sci. USA 95:14470-14475. 